\title{
Comparison of RANS, DES and DDES results for ONERA M-6 Wing at transonic flow speed using an in-house parallel code
}

\author{
Naveed Durrani ${ }^{1}$ and Ning Qin ${ }^{2}$ \\ Department of Mechanical Engineering, University of Sheffield, UK
}

\begin{abstract}
The very first thought that comes to the mind with the application area of the DES and DDES schemes is a massively separated flow with highly unsteady flowfield. However, for various complex three dimensional cases, there is no prior knowledge of the flowfield in the domain and it may have mild separation or no separation at all with a steady domain. This study is carried out to see that what will be the behaviour of the DES and DDES schemes in comparison with the URANS scheme if they are applied to a steady state case. An in-house mpi code DG-DES is used for the present study. Three different flux computational schemes named Roe, AUSM and HLLC schemes within DES formulation are compared to check the response for the flows without massive separation and unsteadiness. The cases are run in both single and double precision mode for DES formulation using Roe flux computational scheme to appreciate the accuracy of the solver.

A good comparison of pressure distribution with the experimental data is obtained for all URANS, DES and DDES simulations. The pressure distribution results for DES scheme using single and double precision agree well with the experimental data. The pressure distribution predicted by DES using Roe, AUSM and HLLC schemes agree well with the experimental data. The computed values of $\mathrm{Cl}$ and $\mathrm{Cd}$ are also in close approximity to the other studies. The drag predicted by all DES and DDES simulations is lower than the URANS scheme. It indicates that the DES and DDES schemes generate lower dissipation due to switching to the LES mode and hence result in lower drag prediction as compared with the URANS solution. There is no anomaly observed in the flow due to the use of DES or DDES for steady flow case.
\end{abstract}

\section{Nomenclature}

$\begin{array}{ll}\mathrm{A} & =\text { surface area } \\ b & =\text { span of the wing } \\ C_{l} & =\text { coefficient of lift } \\ \mathrm{C}_{\mathrm{d}} & =\text { coefficient of drag } \\ C_{p} & =\text { pressure coefficient } \\ \mathrm{d} t & =\text { time step } \\ \mathrm{F} & =\text { Inviscid flux vector } \\ \mathrm{f} & =\text { shedding frequency } \\ \mathrm{G} & =\text { Viscous flux vector } \\ \mathrm{Q} & =\text { Primitive variables vector } \\ \mathrm{Re} & =\text { Reynolds number } \\ \mathrm{S} & =\text { Strain rate tensor } \\ \mathrm{U}_{\mathrm{inf}} & =\text { Free stream velocity } \\ \mathrm{V} & =\text { Volume } \\ \mathrm{W} & =\text { Conserved variables vector } \\ \tilde{v} & =\text { modified kinematic eddy viscosity }\end{array}$

\footnotetext{
${ }^{1}$ Post Graduate Student, Department of Mechanical Engineering, AIAA member

${ }^{2}$ Professor of Aerodynamics, Department of Mechanical Engineering, AIAA Associate
} 


$$
\begin{array}{ll}
v_{T} & =\text { kinematic eddy viscosity } \\
\mu_{T} & =
\end{array}
$$

\section{Acronyms:}

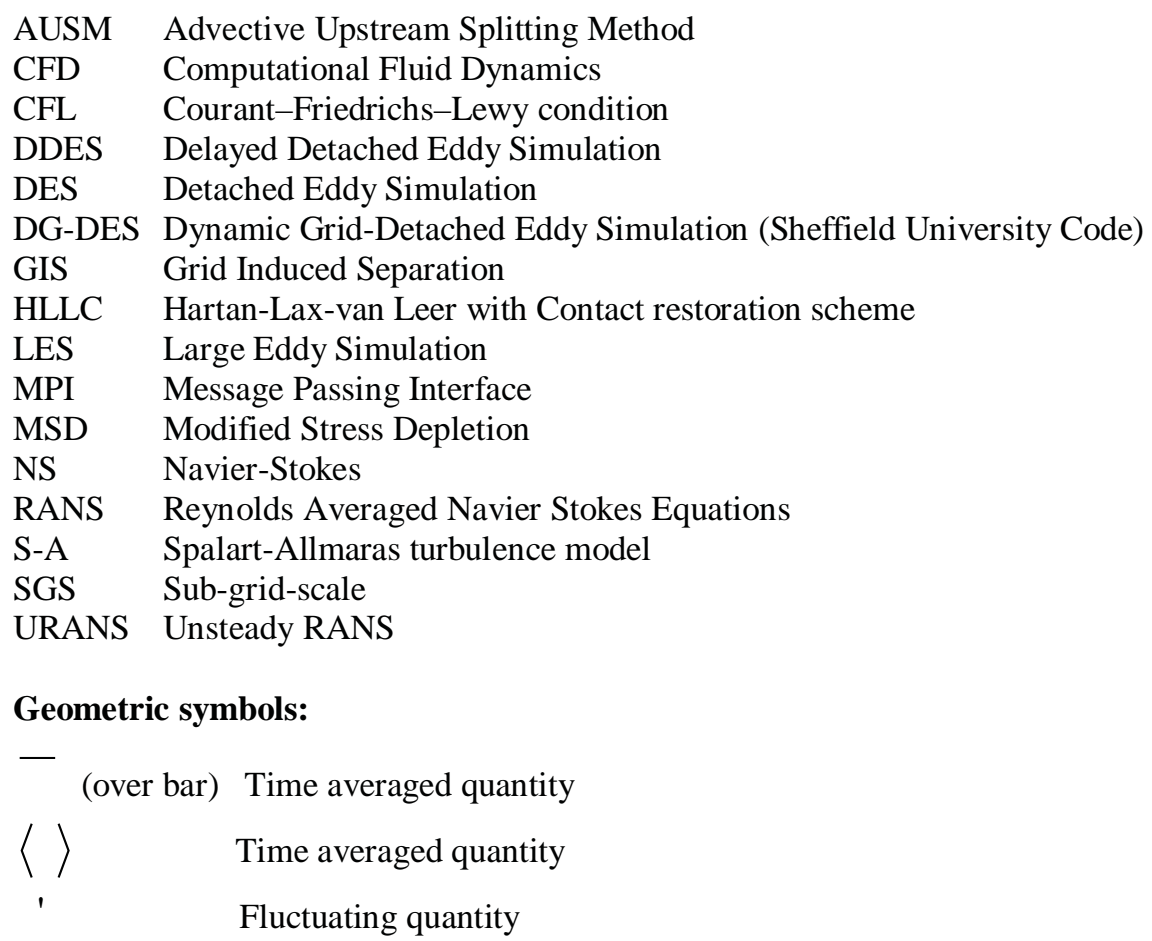

\section{Introduction}

$\mathrm{H}$ YBRID RANS-LES methods have gained popularity amongst a sizeable CFD community due to their simplicity, lower computational cost and ability to capture massively separated flows. Detached-Eddy simulation $^{1}$ (DES) is one such method that has gained considerable attention and popularity prompting its application in various studies ${ }^{3-11,14}$. The original DES $^{1}$ (now termed as DES97) was non-zonal in nature and was aimed for the massively separated flows. It was aimed to solve the entire boundary layer in RANS mode and rest of the domain including the separation zone in the LES mode. However, with the surge in application of this methodology some problems were highlighted. One problem was the inehernet short-coming in the original methodology to switch from RANS to LES mode for the thick boundary layers leading to separation. This problem caused the switching to LES within the boundary layer leading to the modeled stress depletion (MSD). Another problem was more to do with the user's improper generation of the mesh for this scheme. So, the problems were faced due to the improper mesh generation or use of the scheme for the areas for which it is not intended for. This lead to its modification by its proponents termed as “delayed detached-eddy simulation” (DDES) ${ }^{2}$. 'Delay' basically meant the delay in switching from RANS to LES in the boundary layer region. Further details about DES and DDES can be found in review in Ref. 12.

As one of the reason for proposing DDES was the use of DES scheme in the cases which were not appropriately suited. For the flows with no massive seaparation or for flows which are not "natural DES flows", it is interesting to see the results of DES and DDES. Present CFD study is carried out using URANS, DES and DDES schemes for the flow simulation over Onera M6 wing and results are compared with the experimental data ${ }^{20}$ and with other numerical studies ${ }^{22-24}$.

\section{Case Setup}

Only half of the wing along the span is considered for the numerical simulation. The Spalart-Allmaras turbulence model $^{13}$ is used for the steady state simulation. It is to be mentioned that the cases are run in unsteady mode for a 
long period of time to achieve the pseudo steady state. The freestream flow conditions are assumed with further case details as mentioned in "Table 1 ".

\begin{tabular}{|c|c|c|c|}
\hline Mach Number & Reynolds Number & Angle of Attack & Side Slip Angle \\
\hline 0.8395 & $11.72 \times 10^{6}$ & 3.06 & 0.0 \\
\hline
\end{tabular}

Table 1 Case setup of Onera M6 wing at transonic speeds

Mesh used in this validation was generated using commercial software Fluent. This mesh has a baseline prismatic layer with tetrahedral elements on top for economizing the mesh density. It is an unstructured grid and further details are presented below in Table 2.

\begin{tabular}{|c|c|c|c|}
\hline Total nodes & Total cells & Tetrahedral & Prism \\
\hline 621,282 & $1,520,491$ & 452,651 & $1,067,840$ \\
\hline
\end{tabular}

Table 2 Mesh size details

\section{A. RANS formulation}

\section{Numerical Scheme}

The favre-averaged NS equation is given as:

$$
\frac{\partial}{\partial t}\left(\bar{\rho} \tilde{u}_{i}\right)+\frac{\partial}{\partial x_{j}}\left(\bar{\rho} \tilde{u}_{i} \tilde{u}_{j}\right)=-\frac{\partial \bar{p}}{\partial x_{i}}+\frac{\partial}{\partial x_{j}}\left(\bar{\tau}_{i j}+\tau_{i j}^{R}\right)
$$

where, $\tau_{i j}^{R}=-\rho \overline{u_{i}^{\prime \prime} u_{j}^{\prime \prime}}=2 \mu_{T} S_{i j}+\lambda_{T} \frac{\partial \tilde{u}_{k}}{\partial x_{k}} \delta_{i j}$

To close the system, the variable $\mu_{T}$ is calculated using the S-A turbulence model ${ }^{13}$.

$$
\frac{\partial \tilde{v}}{\partial t}+\nabla \cdot\left[\tilde{v} \mathbf{v}-\frac{1}{\sigma}(v+\tilde{v}) \nabla \tilde{v}\right]=\left(c_{b 1} \tilde{S}+\nabla \cdot \mathbf{v}\right) \tilde{v}-c_{w 1} f_{w}\left(\frac{\tilde{v}}{d}\right)^{2}+\frac{c_{b 2}}{\sigma}(\nabla \tilde{v})^{2}
$$

Where, all the terms on the right hand side are called the 'source terms' denoted by 'src'.

$$
\frac{\partial \tilde{v}}{\partial t}+\nabla \cdot\left[\tilde{v} \mathbf{v}-\frac{1}{\sigma}(v+\tilde{v}) \nabla \tilde{v}\right]=s r c
$$

The above equation can be re-organized to display the various terms in more physically descriptive fashion with various contributing terms as follows:

$$
\begin{gathered}
\underbrace{\frac{1}{\sigma}\left[\nabla \cdot(v(+\tilde{v}) \nabla \tilde{v})+c_{b 2}(\nabla \tilde{v})^{2}\right]}_{\text {DiffusionTerm }} ; \underbrace{c_{b 1} \tilde{S} \tilde{v}-c_{b 1} f_{v 2} \tilde{S} \tilde{v}}_{\text {Production Term }} ; f_{v 2}=1-\frac{\chi}{1+\chi f_{v 1}} \\
\underbrace{c_{w 1} f_{w}\left(\frac{\tilde{v}}{d}\right)^{2}-\frac{c_{b 1}}{\kappa^{2}}\left(\frac{\tilde{v}}{d}\right)^{2}}_{\text {Destruction Term }} \chi=\frac{\tilde{v}}{v}, \quad f_{v 1}=\frac{\chi^{3}}{\chi^{3}+C_{v 1}^{3}}
\end{gathered}
$$

In the regions, away from the wall, the production of the turbulent viscosity is equal to the destruction term. 


$$
\underbrace{c_{b 1} \tilde{S} \tilde{v}-c_{b 1} f_{v 2} \tilde{S} \tilde{v}}_{\text {Production Term }} \approx \underbrace{c_{w 1} f_{w}\left(\frac{\tilde{v}}{d}\right)^{2}-\frac{c_{b 1}}{\kappa^{2}}\left(\frac{\tilde{v}}{d}\right)^{2}}_{\text {Destruction Term }}
$$

This gives the relationship $\tilde{v} \alpha \tilde{S} d^{2}$

$$
\operatorname{Or} \mu_{T} \alpha \tilde{S} d^{2} \quad \because \tilde{v}=\frac{\mu_{T}}{\rho f_{v 1}}
$$

Where, $\mathrm{d}$ is the distance to the closest wall.

\section{B. LES formulation}

The filtered momentum equation for LES is given as

$$
\frac{\partial}{\partial t}\left(\rho \bar{u}_{i}\right)+\frac{\partial}{\partial x_{j}}\left(\rho \bar{u}_{i} \bar{u}_{j}\right)=-\frac{\partial \overline{p^{*}}}{\partial x_{i}}+\frac{\partial}{\partial x_{j}}\left[2\left(\mu+\mu_{R}\right) \bar{S}_{i j}\right]
$$

where, for the Smagorinsky model $\mu_{R}=\rho C_{S}^{2} L^{2}\left(2 \bar{S}_{i j} \bar{S}_{i j}\right)^{1 / 2}$

$$
\text { Or } \mu_{R} \alpha \bar{S}_{i j} L^{2}
$$

$L$ is the width of the filter.

The space filtered momentum equation for LES "Eq. 4-a" looks remarkably similar to the momentum equation of the RANS formulation described by "Eq. 3-a". By observing the spatial filtering for LES, it is evident that the effect of the filtering is to introduce fictitious stresses termed as "residual stresses". These residual stresses are similar to the Reynolds stresses introduced by the time-averaging. In short, the structure of the RANS and LES equations in the region away from wall is quite similar. This similarity holds away from the wall because in this region, the production and destruction terms of the S-A turbulence model for RANS balance each other and the resultant set of equations is similar to space filtered LES equations using Smagorinsky's model.

\section{Detached-eddy simulation (DES)}

The Detached-eddy Simulation approach (DES) was proposed by Spalart et al. in $1997^{11}$. The main theme of the DES scheme was to combine the strengths of the RANS scheme near the solid wall boundaries and of LES elsewhere. The natural implementation was intended to simulate the entire boundary layer using the RANS and separated region with the LES. It was aimed primarily for the cases in which the flow develops a sudden unsteadiness due to a blunt body or sharp edge with the generation of huge downstream turbulence, independent of upstream turbulence history.

The modification from RANS to DES is quite simple. The only change lies in the length scale, d. It is replaced for the DES as the minimum between the distance to the wall and a length proportional to the local grid spacing. It is represented mathematically as

$$
d_{\mathrm{DES}}=\min \left(d, C_{\mathrm{DES}} \Delta\right)
$$

where, $C_{\mathrm{DES}}$ represents a model constant taken as 0.65 in different studies ${ }^{14} . \Delta$ is the local grid spacing.

\section{Delayed Detached-eddy simulation (DDES)}

As discussed in the introduction, with the expanding application of DES, some of its weaknesses were also exposed. For the original DES, it is imperative to use mesh which is in conjunction with the natural DES architecture. Some applications areas, in which the natural theme of DES is difficult to maintain, were found to cause malfunctioning of the DES simulation. Noticeably, the flow conditions involving thick boundary layer may be difficult to be tackled just on the basis of distance parameter, $d$, only. It may force the solver to switch to the LES 
part in the outer boundary layer region, causing sudden decrease in the modelled stresses, giving rise to separation which is not physical but due to the grid. This problem is termed as the Grid Induced Separation (GIS) and this erratic phenomenon is termed as modelled stress depletion (MSD). Menter and Kuntz ${ }^{15}$ were the first to highlight this problem. This issue was addressed by proposing a fix to this problem in the form of delayed DES (DDES) ${ }^{2}$, similar to the Menter's scaling functions in his SST turbulence model ${ }^{25}$. The proponents of the DES scheme renamed the original DES to DES97 to indicate the initial DES scheme proposed in 1997.

The main idea of DDES is to include the molecular and turbulent viscosity information into the switching mechanism to delay the premature switching in the boundary layer region.

$$
\begin{gathered}
\tilde{d} \equiv d-f_{d} \max \left(0, \mathrm{~d}-\mathrm{C}_{D E S} \Delta\right) \\
f_{d} \equiv 1-\tanh \left(\left[8 r_{d}\right]^{3}\right) \\
r_{d} \equiv \frac{v_{t}+v}{\sqrt{U_{i, j} U_{i, j}} \kappa^{2} d^{2}}
\end{gathered}
$$

Further details of this modification can be found in Ref. 2.

\section{Numerical solution details}

An in-house parallel, density based unstructured solver called Dynamic Grid Detached-eddy Simulation (DGDES ${ }^{16,17}$ is used for the present study. The code solves the unsteady Reynolds-Averaged Navier-Stokes (RANS) equations using dual-time stepping. These governing equations are discretized using a cell-centered finite volume method. The convective terms are discretized using the Roe scheme ${ }^{18}$. A single equation Spalart-Allmaras turbulence model is used for the Detached-eddy simulation formulation. Metis ${ }^{19}$, an open source program is used for the mesh partitioning in the DG-DES code for parallel computing. Master node does not take part directly in the computations but manages the overall slave node computations.The $2^{\text {nd }}$ order spatial accuracy is achieved with the piecewise linear reconstruction of the flow variables. The higher spatial accuracy presents better flow field results. Present simulations are run in full turbulent mode (no transition or trip functions used).

\section{Results}

Figure 1(b) indicates the mesh at the symmetry axis with M6-Wing surface coloured with pressure contours. The wing tip effects towards the trailing edge are very clear. The right side figure is $\mathrm{Y}+$ plot on the surface of the M6 Wing. $\mathrm{Y}+$ is less than 1 almost everywhere on the surface. Only in a small region with shock on the suction side has $\mathrm{Y}+$ value increasing to around 3 . The subsequent results are in very good agreement with the experimental data. Figure 2 indicates the Mach number plot in 'line mode' at the symmetry axis and the wing surface is colured with the static pressure contours. The " $\lambda$-shock", as termed generally in literature, can be observed in Fig. 2 on the wing surface from pressure plot and in Mach plot as well. In addition, the maximum Mach number obtained by the CFD simulation is around 1.14 which is in accordance with the experimental observations ${ }^{20}$ and other simulation results ${ }^{21}$. The surface plot indicates the maximum static pressure at the leading edge corresponding to stagnation pressure and a clear demarcation of high and low pressure regions is visible across the shock wave. Down towards the wing tip in spanwise direction, the increase in low pressure region can be observed. It represents the strong tip effect and spanwise three dimensional flow effects. The two dimensional simulation is unable to capture this and it is very important in real life finite-span simulations. 

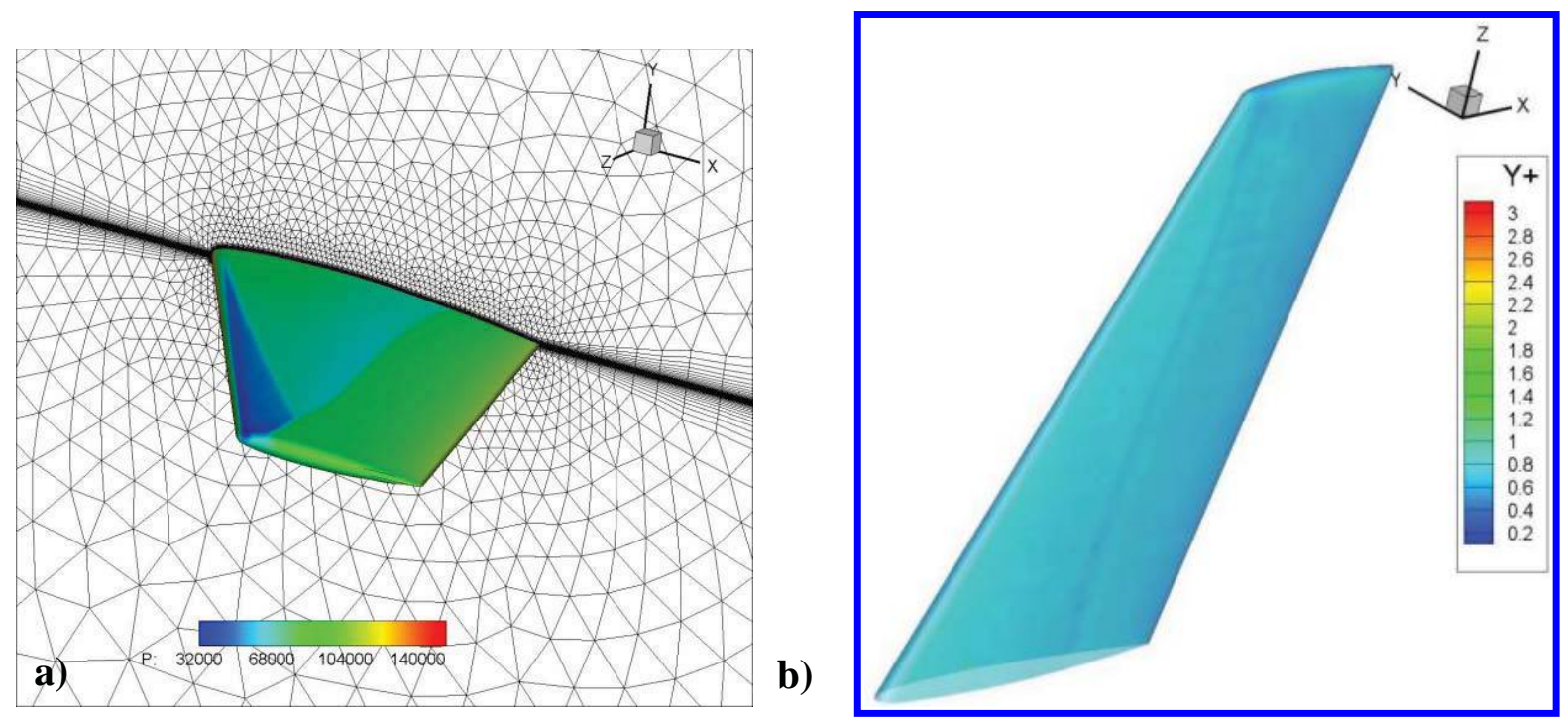

Figure1. a) Mesh along the symmetry plane with M6 Wing surface colour with pressure contour. b) M6 Wing surface colour with $\mathrm{Y}^{+}$.

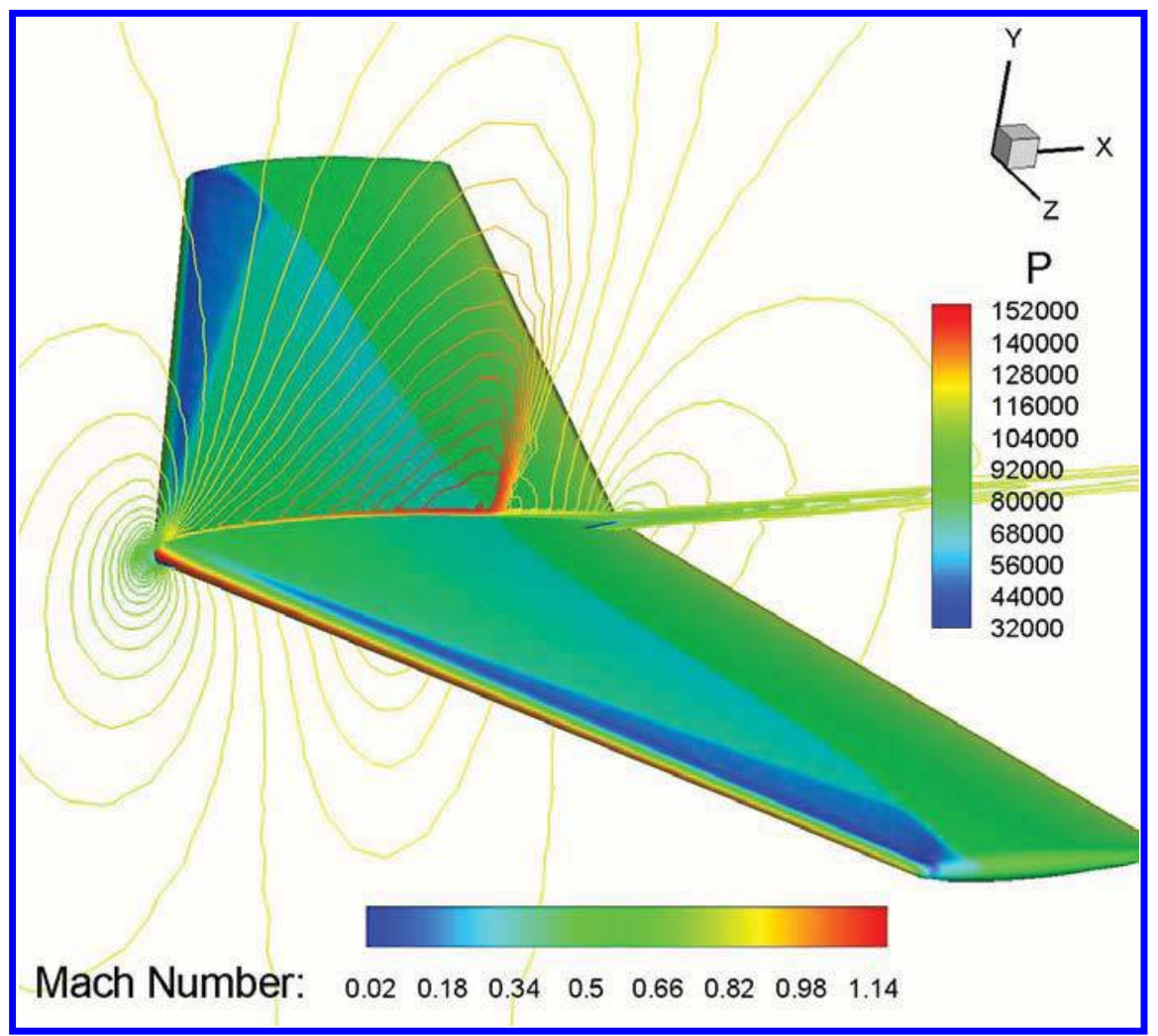

Figure 2. Onera M6 wing surface pressure plot (flood mode) and symmetry plane Mach number nlot (line mode) 


\begin{tabular}{|c|c|c|}
\hline Plot & Spanwise Location ( $\eta$ ) y/b & Actual Height (m) \\
\hline Cp1 & $0.2(20 \%)$ & 0.227 \\
\hline Cp2 & $0.44(44 \%)$ & 0.4994 \\
\hline Cp3 & $0.65(65 \%)$ & 0.73775 \\
\hline Cp4 & $0.8 \quad(80 \%)$ & 0.908 \\
\hline Cp5 & $0.9 \quad(90 \%)$ & 1.0215 \\
\hline Cp6 & $0.95(95 \%)$ & 1.07825 \\
\hline
\end{tabular}

Table 3. Span wise location for surface Cp comparison

The results of pressure distribution for the negative $C_{p}$ along different spanwise locations as tabulated in Table 3 , are shown in Fig. 3 and Fig. 4.

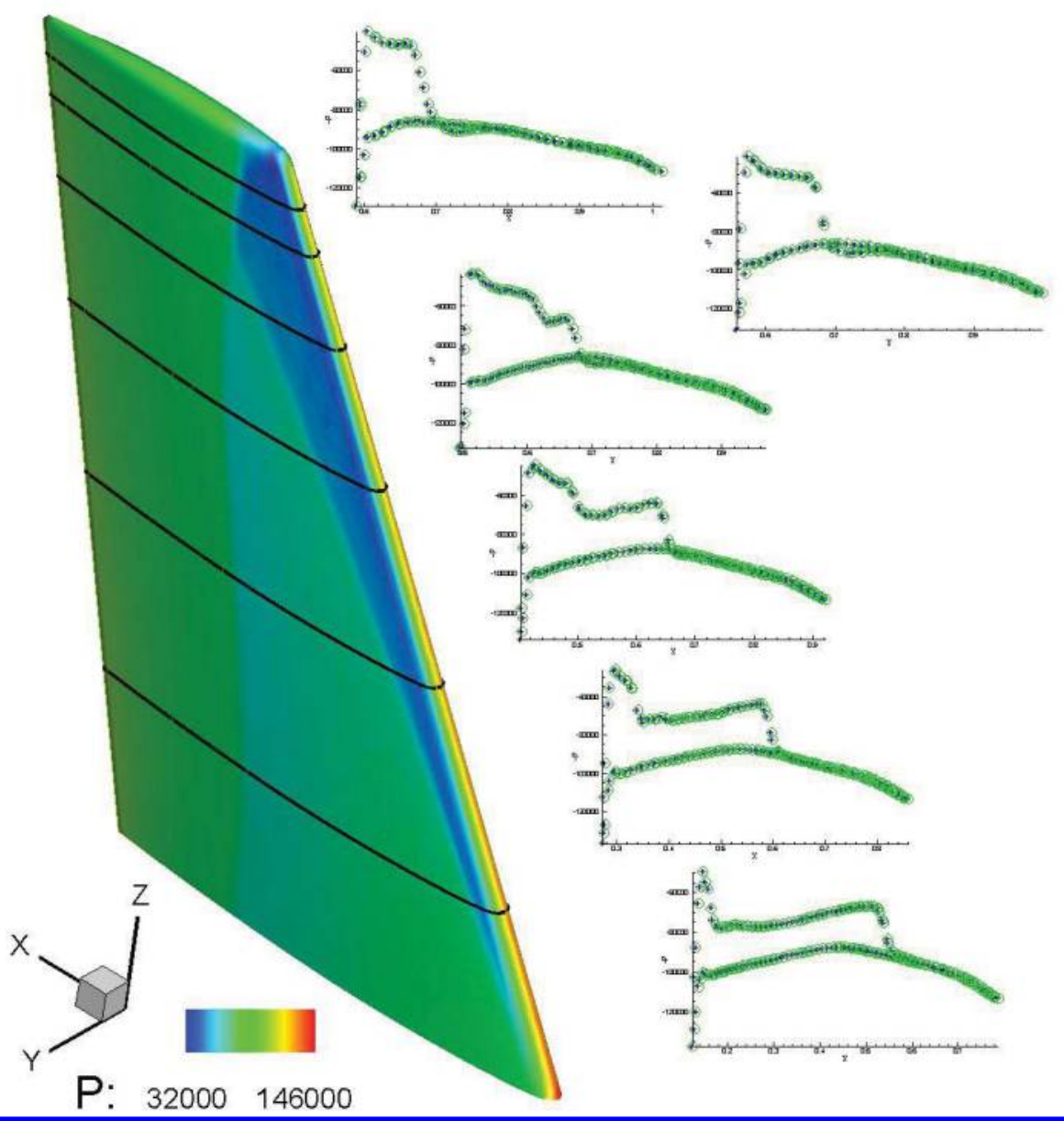

Figure 3. Onera M6 wing comparisons of pressure coefficient results from single and double precision solver using DES (Roe scheme based) at different spanwise locations. Single precision results are represented with "+” sign and double precision with a green colour circle.

Figure 3. indicates a comparison of negative pressure coefficient computed using single and double precision DES solver. Further detailed analysis was done using different inviscid flux computation options implemented in the code including Roe, AUSM and HLLC schemes. 
The resultant $C_{p}$ values are plotted with experimental data for comparison in Fig 4 a-f. Experiments were conducted on M6 wing at transonic flow conditions by Schmitt et $\mathrm{al}^{20}$.

Experimental surface pressure distribution at different spanwise stations is available and is compared with the numerically simulated results. It can be seen that generally the shock capturing is good and the location of the shock wave is correctly predicted. In figure 4-a, the shock wave is relatively less sharp and its location and resolution is not as well predicted as at other location; but as the shock wave becomes steeper along subsequent cross sections, its location and resolution improves. Overall comparison with the experimental data is good.

One important phenomenon to mention from figure 4a-d is the presence of double shock wave on the suction side of the M6 Wing along the chordwise direction. It is typical of " $\lambda$-shock". Going down towards the wing tip in the spanwise direction, the distance between the double shocks keeps reducing until it merges to a single shock after around $85 \%$ of spanwise direction (as shown in $90 \%$ spanwise $C_{p}$ plot as a single shock). This region where the shocks merge, makes it difficult to obtain good $\mathrm{C}_{\mathrm{p}}$ at $80 \%$ spanwise location in comparison with the experimental data. It is interesting to mention that famous CFD solver WIND failed to predict the presence of double shock wave at $80 \%$ spanwise location ${ }^{21}$, however, it is well predicted in present simulation.

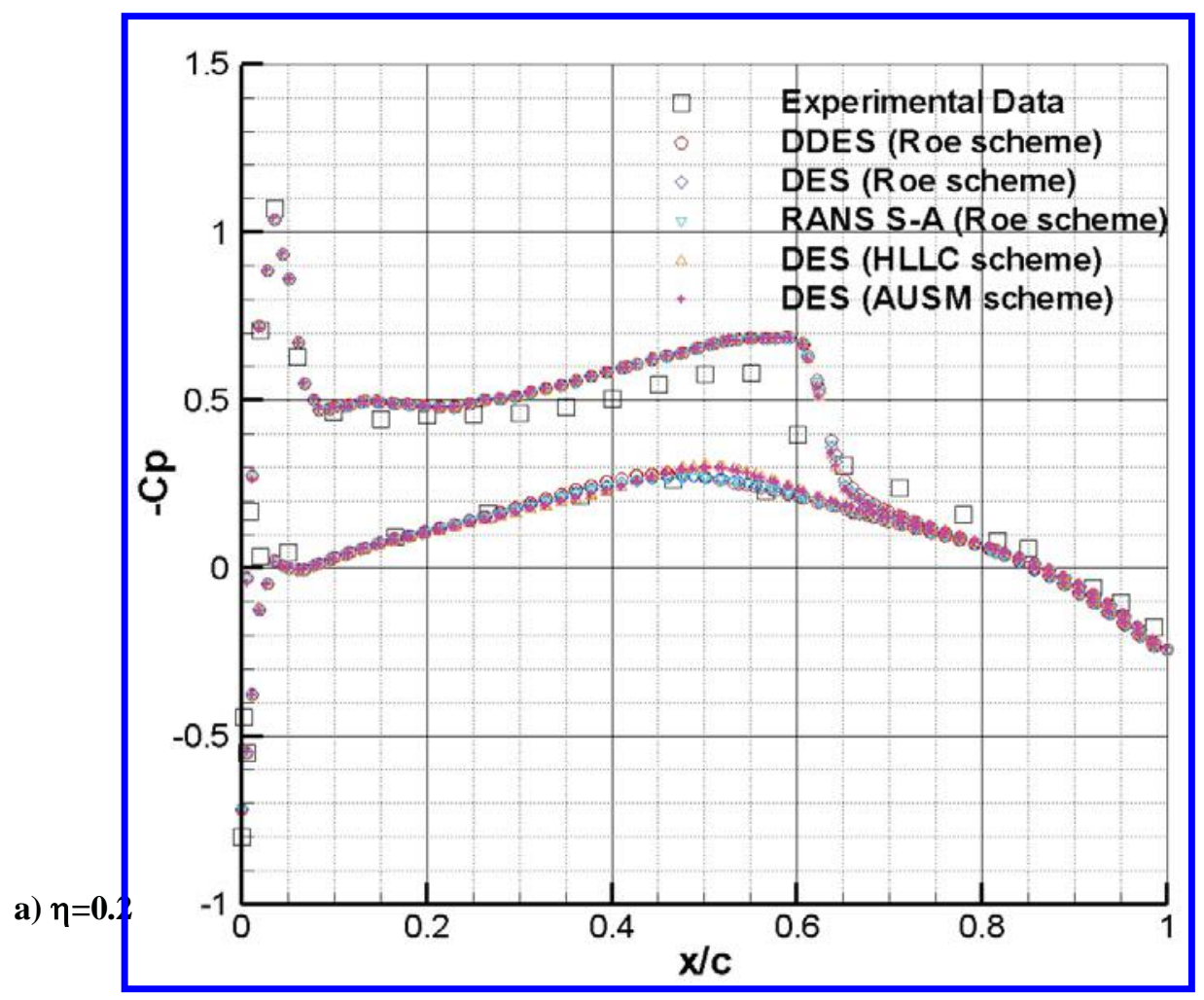



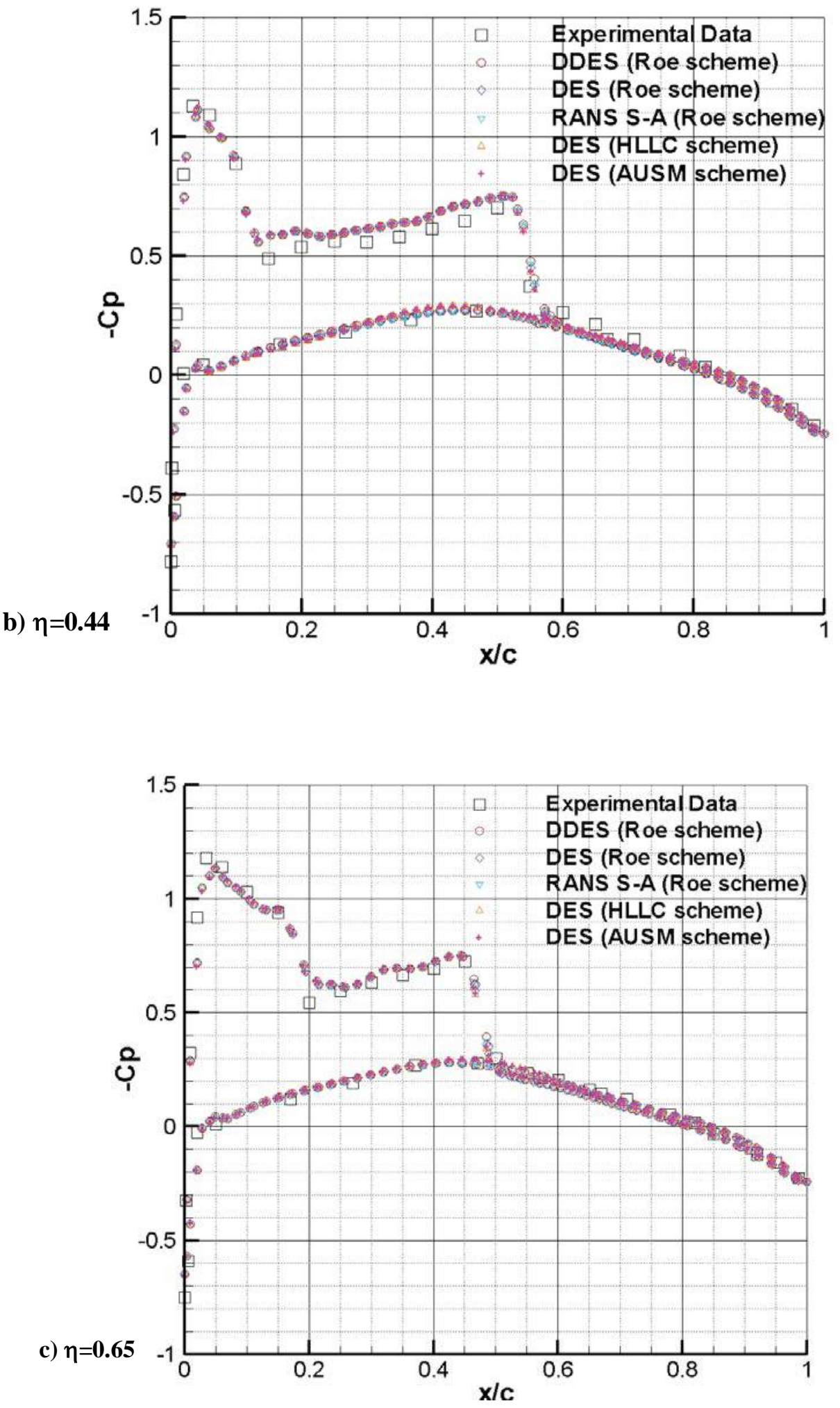

American Institute of Aeronautics and Astronautics 


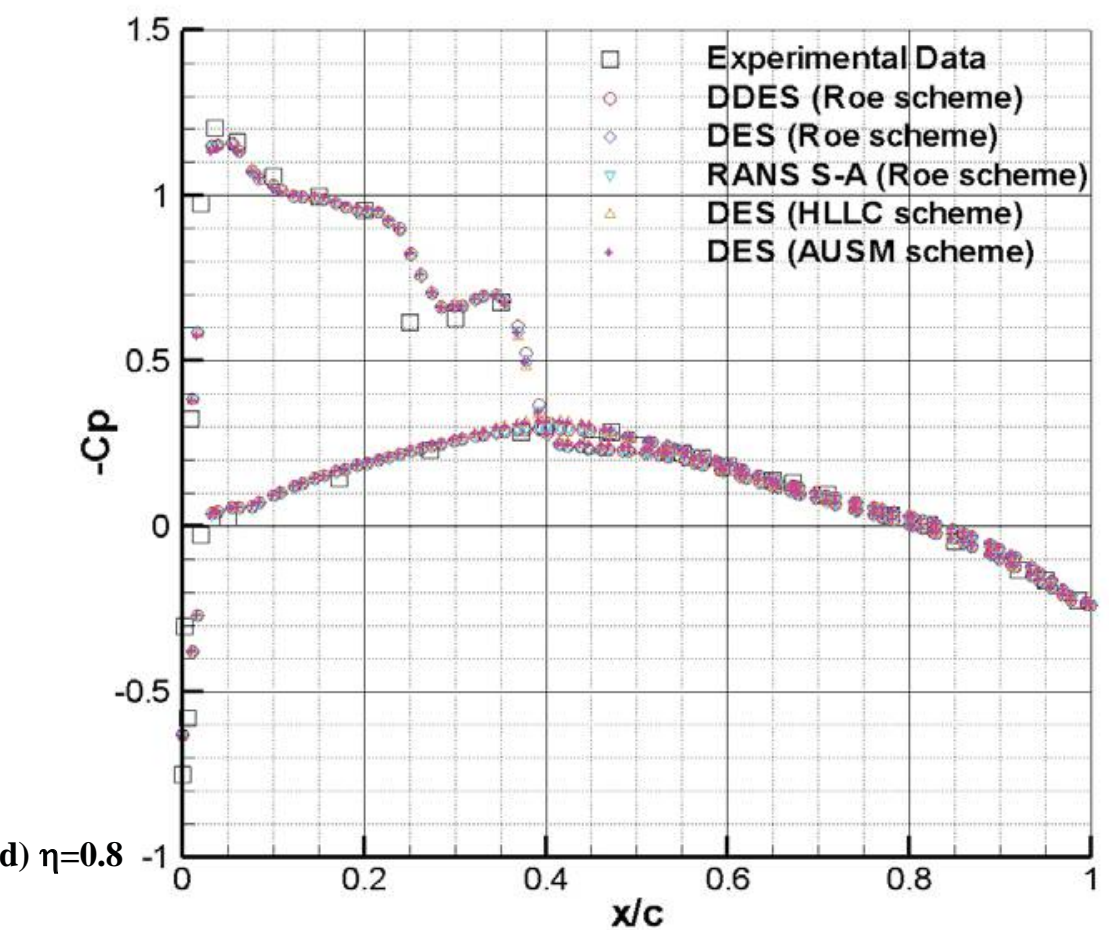

e) $\eta=0.9$

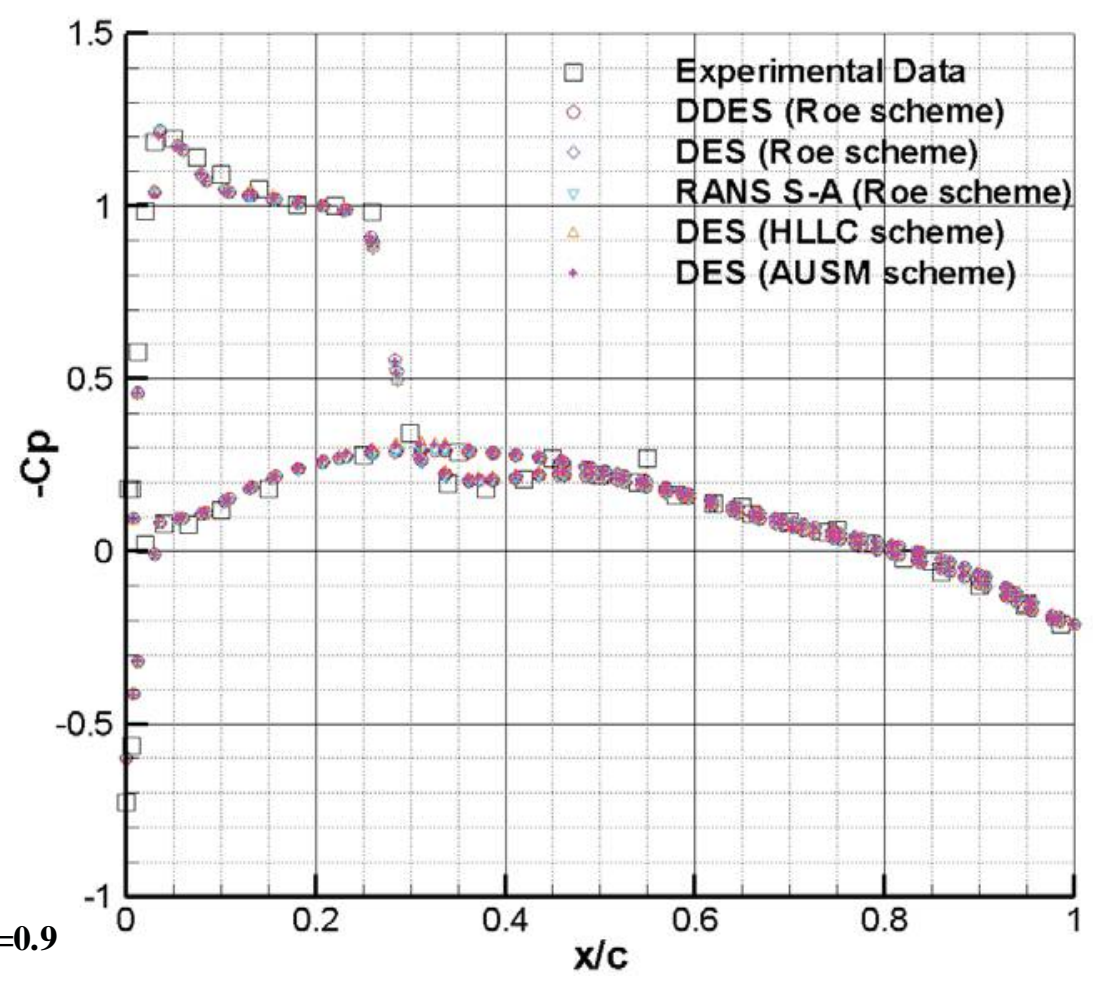




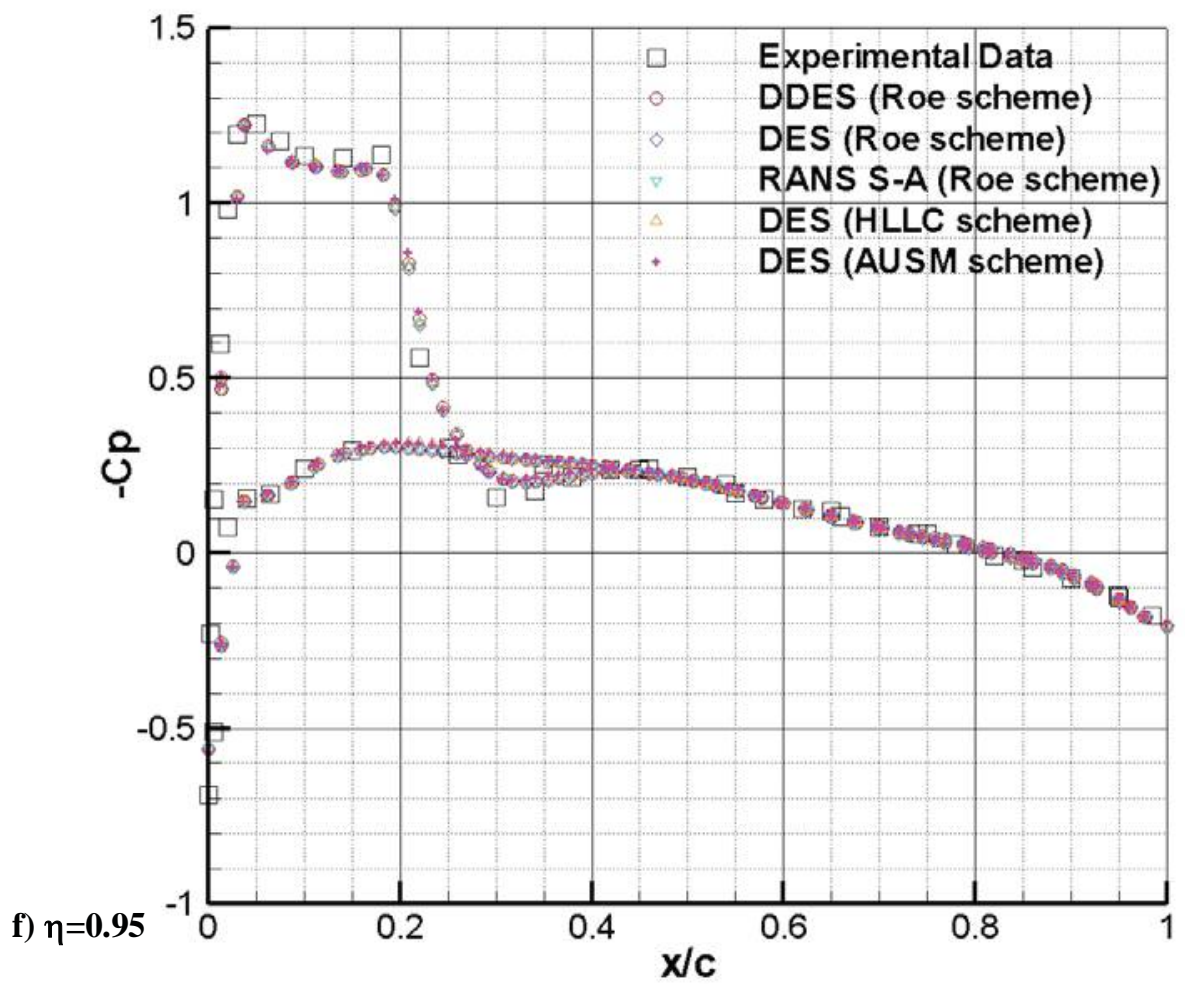

Figure 4. (a-f) Surface Cp comparison of different turbulent flow simulation schemes at different spanwise locations $(\eta)$ with experimental data

The table 4 presents the results comparison from different numerical simulations. It can be seen that the coefficient of lift and drag computed by using the DG-DES are in good agreement with the other simulations.

Overall the comparison is quite good. The lift and drag coefficients $\left(C_{l}\right.$ and $\left.C_{d}\right)$ values obtained are quite encouraging. The experimental data for $C_{l}$ and $C_{d}$ was not found and hence the results were compared with the other studies as presented in the Table 4.

\begin{tabular}{|c|c|c|c|c|c|c|}
\hline Solver & $\begin{array}{c}\text { Turb. } \\
\text { Model }\end{array}$ & $\begin{array}{c}\text { Flux } \\
\text { Scheme }\end{array}$ & Precision & Mesh Type & $\mathbf{C}_{\mathbf{l}}$ & $\mathbf{C}_{\mathbf{d}}$ \\
\hline DG-DES & S-A & Roe & Double & Unstructured & 0.2579 & 0.01970 \\
\hline DG-DES & DES & Roe & Double & Unstructured & 0.2564 & 0.01960 \\
\hline DG-DES & DDES & Roe & Double & Unstructured & 0.2580 & 0.01955 \\
\hline DG-DES & DES & AUSM & Double & Unstructured & 0.2540 & 0.01910 \\
\hline DG-DES & DES & HLLC & Double & Unstructured & 0.2550 & 0.01991 \\
\hline MERLIN ${ }^{22}$ & S-A & Osher & Double & Structured & 0.2697 & 0.01736 \\
\hline Neilsen et al. $^{24}$ & S-A & $\mathrm{xx}$ & $\mathrm{xx}$ & Unstructured & 0.2530 & 0.01680 \\
\hline Lee et. al. $^{23}$ & SST & $\mathrm{xx}$ & $\mathrm{xx}$ & Structured & 0.2622 & 0.01751 \\
\hline
\end{tabular}

Table 4. Comparison of different mesh parameters using DG-DES solve with different options with other studies

11

American Institute of Aeronautics and Astronautics 
All these studies also compared the $C_{l}$ and $C_{d}$ with other numerical simulations only and pressure coefficient $\left(C_{p}\right)$ values were compared with the experimental data. The DG-DES results presented for comparison in the table 4 are all with double precision setting.

\section{Conclusion}

The simulations for Onera M6 wing were quite successful and good agreement of the results with other studies and the experimental data was obtained. The close matching of the simulations using the double precision solver and various numerical schemes available in the DG-DES, validated the implementation and accuracy of the code. The good agreement of the $C_{p}$ distribution at different spanwise locations with the experimental data is quite encouraging. Another important observation is that for these high speed flows with no separation, the generated boundary layer is very thin. This thin boundary layer without separation generates the results which are similar for URANS, DES and DDES. It is evident that for such type of cases, both the DES and DDES rely greatly on the underlying RANS results in the near wall region. Drag predicted by the URANS is highest amongst all the other simulations. This justifies the fact that all the DES and DDES computations are using LES mode in the 'farfield' or 'away from the wall' region. The LES mode is expected to give lower dissipation and hence lower drag. No anomaly is observed in results for any case using DES or DDES schemes and all results match well with the other numerical studies. Hence, it can be concluded that for simple steady-state cases or for unsteady cases involving the large steady-state regions, DES and DDES simulations will give URANS like results with lower dissipation.

\section{Acknowledgments}

The first author is funded by the overseas research scholarship (ORS) from the University of Sheffield, UK and is thankfully acknowledged. The simulations were carried out on two of the clusters; "Bluegrid" and "Greengrid" of the Thermofluids Group in the Department of Mechanical Engineering, University of Sheffield.

\section{References}

${ }^{1}$ Spalart, P. R., Jou W-H., Strelets M., Allmaras, S. R., “Comments on the Feasibility of LES for Wings and on a Hybrid RANS/LES Approach” Advances in DNS/LES, $1^{\text {st }}$ AFOSR Int. Conf. on DNS/LES, Greyden Press, Columbus Oh, 4-8 August, 1997.

${ }^{2}$ Spalart, P., Deck, S., Shur, M., Squires, K., Strelets, M. K., and Travin, A., “A New Version of Detached-Eddy Simulation, Resistant to Ambiguous Grid Densities,” Theoretical and Computational Fluid Dynamics. 0935-4964, pp.181-195, July 2006.

${ }^{3}$ Squires, K. D., Krishnan, V., and Forsythe, J. R., "Prediction of the flow over a circular cylinder at high Reynolds number using detached-eddy simulation”, Journal of wind engineering and industrial aerodynamics. pp. 1528-1536, 2008.

${ }^{4}$ J.R. Forsythe, K. D. Squires, K.E. Wurtzler, P.R. Spalart, “Detached-Eddy Simulation of Fighter Aircraft at High Alpha”, AIAA paper, 2002-0591, January 2002.

${ }^{5}$ K. D. Squires, J. R. Forsythe, S. A. Morton, D. C. Blake, M. Serrano, K.E. Wurtzler, W. Z. Strang, R.F. Tomaro, P.R. Spalart, "Analysis of Full Aircraft with Massive Seperation using Detached-Eddy Simulation” Computational Fluid Dynamics Journal ISCFD Japan, Vol. 13, No. 3, January, 2005.

${ }^{6}$ S.A. Morton, J.R. Forsythe, K.D, A. Mithchell, D. Hajek, “DES and RANS” Simulation of Delta Wing Vortical Flow”, AIAA paper, 2002-0587, 2002.

${ }^{7}$ K. D. Squires, J. R. Forsythe, S. A. Morton, W. Z. Strang, K.E. Wurtzler, R.F. Tomaro, M.J. Grismer, P.R. Spalart, "Progress on Detached-Eddy Simulation of Massively Seperated Flows”, AIAA paper, 2002-1021, January 2002.

${ }^{8}$ S. A. Morton, J. R. Forsythe, K. D. Squires, K.E. Wurtzler, "Assessment of Unstructured Grids for Detached-Eddy Simulation of High Reynolds Number Separated Flows.”, 8 ISGG Conference, Honolulu, June,2002.

${ }^{9}$ M. Strelets, "Detached Eddy Simulation of Massively Separated Flows“ AIAA Paper 2001-0879, January 2001.

${ }^{10}$ K. D. Squires, J. R. Forsythe, S. A. Morton, M. J. Grismer, and P. R. Spalart, "Progress on Detached-Eddy Simulation of Massively Separated Flows“, AIAA Paper 2002-1021, 2002.

${ }^{11}$ P. R. Spalart, "Young-Person’s Guide to Detached-Eddy Simulation Grids“, NASA/CR-2001-211031, pp. 23 , July 2001.

${ }^{12}$ P. R. Spalart, ”Detached-Eddy Simulation”, Annu. Rev. Fluid Mech. 41:181-202, 2009.

${ }^{13}$ P. R. Spalart and S. R. Allmaras, “A One-Equation Turbulence Model for Aerodynamic Flows“, AIAA Paper 92-0439, January 1992.

${ }^{14}$ M. Shur, P.R. Spalart, M. Strelets and A. Travin, “Detached-eddy simulation of an airfoil at high angle of attack”, 1999. In: Proceedingsof the 4th International Symposium on Engineering Turbulence Modelling and Measurements, Corsica. 24-26 May, Elsevier, Amsterdam, pp. 669-678.

${ }^{15}$ Menter, F.R., Kuntz, M, “Adaptation of Eddy Viscosity Turbulence Models to Unsteady Separated Flow Behind Vehicles.” In: McCallen, R., Browand, F., Ross, J., (eds.) Symposium on "the aerodynamics of heavy vehicles: trucks, busses, and trains” Monterey, USA, 2-6 Dec 2002, Springer, Berlin Heidelberg New York, 2004.

${ }^{16}$ N. I. Durrani, "Hybrid RANS-LES Simulations for Separated Flows Using Dynamic Grids”, Ph. D. thesis, University of Sheffield, UK, March 2009.

${ }^{17}$ N. Qin and H. Xia, "Detached eddy simulation of a synthetic jet for flow control”, Proceedings of the Institution of Mechanical Engineers, Part I: Journal of Systems and Control Engineering, Vol. 222, No. 5, pp. 373-380. 
${ }^{18}$ P. L. Roe, “Approximate Riemann Solvers, Parameters Vectors and Difference Schemes“, Journal of Computational Physics, 43:357-372, 1981.

${ }^{19}$ G. Karypis and V. Kumar, "User Manual of METIS: A Software Package for Partitioning Unstructured Graphs, Partitioning Meshes and Computing Fill-Reduced Orderings of Sparse Matrices, Version 4.0“, University of Minnesota, 1998.

${ }^{20} \mathrm{~V}$. Schmitt and F. Charpin, "Pressure Distribution on the ONERA-M6-Wing at Transonic Mach Numbers“, In Experimental Data Base for Computer Program Assessment. Report of the Fluid Dynamics Panel Working Group 04, AGARD AR 138, May 1979.

${ }^{21}$ J. W. Slater. ONERA M6 Wing: Study 1. NPARC Alliance Validation Archive, http://www.grc.nasa.gov/WWW/wind/valid/m6wing/m6wing01/m6wing01.html, 2002.

${ }^{22}$ Le Moigne, "A Discrete Navier-Stokes Adjoint Method for Aerodynamic Optimization of Blended Wing-Body Configurations“, PhD thesis, Cranfield University, United Kingdom, 2002.

${ }^{23}$ J. Lee, C. S. Kim, C. Kim, O. H. Rho, and K. D. Lee, "Parallelized Design Optimization for Transonic Wings using Aerodynamic Sensitivity Analysis“, AIAA Paper 2002-0264, 2002.

${ }^{24}$ E. J. Neilsen and W. K. Anderson, "Recent Improvements in Aerodynamic Design Optimization on Unstructured Meshes“, AIAA Paper 2001-0596, 2001.

${ }^{25}$ Menter, F. R. (1994), "Two-Equation Eddy-Viscosity Turbulence Models for Engineering Applications", AIAA Journal, vol. 32, pp. 269-289 